\title{
Modal description of internal optimal streaks
}

\author{
MARIA HIGUERA AND JOSÉ M. VEGA \\ E. T. S. Ingenieros Aeronáuticos. Universidad Politécnica de Madrid
}

This paper deals with the definition and description of optimal streaky (S) perturbations in a Blasius boundary layer. First, the asymptotic behaviours of S-perturbations near the free stream and the leading edge are studied to conclude that the former is slaved to the solution inside the boundary layer. Based on these results, a quite precise numerical scheme is constructed that allows concluding that S-perturbations produced inside the boundary layer, near the leading edge, can be defined in terms of just one streamwise-evolving solution of the linearized equations, associated with the first eigenmode of an eigenvalue problem first formulated by Luchini (J. Fluid Mech., vol. 327, 1996, p. 101). Such solution may be seen as an internal unstable streaky mode of the boundary layer, similar to eigenmodes of linearized stability problems. The remaining modes decay streamwise. Thus, the definition of streaks in terms of an optimization problem that is used nowadays is not necessary.

\section{Introduction}

Streaky perturbations of two-dimensional boundary layers are three-dimensional waves that show a short-wave spanwise oscillation and a slower streamwise evolution, with characteristic lengths comparable to the boundary layer thickness and to distance to the leading edge, respectively. This is in contrast to Tollmien-Schlichting (TS) modes, which show a short wave streamwise oscillation. S-perturbations were already recognized by Crow (1966) and subsequently analysed by Ellingsen \& Palm (1975), Landhal (1980), Luchini (1996, 2000), Andersson, Berggren \& Henningson (1999) and Leib, Wundrow \& Goldstein (1999) among others. These are somehow the (mathematically) natural perturbations of the boundary layer since they exhibit the same scaling as the steady state and thus admit a Reynolds-number-independent formulation, while Reynolds number cannot be eliminated from the Orr-Sommerfield equation that governs TS-modes. Streamwise evolution of S-perturbations first shows algebraic growth and then exponential decay due to viscous dissipation. Such growth-decay combination, known as transient growth (Hultgren \& Gustavsson 1981; Threfethen et al. 1993; Bagget \& Threfethen 1997; Schmid 2007), can still be dangerous if algebraic growth is large enough as to enhance nonlinear interaction, which may allow a three-dimensional destabilization of TS-modes by S-perturbations (Klebanoff, Tidstrom \& Sargent 1962) and promote bypass transition (Morkovin 1984; Morkovin \& Reshotko 1990). S-perturbations, on the other hand, may also have a beneficial effect for smaller (than those promoting bypass transition) amplitudes, as shown by Cossu \& Brandt (2002) (see also Fransson et al. 2004, 2006). 

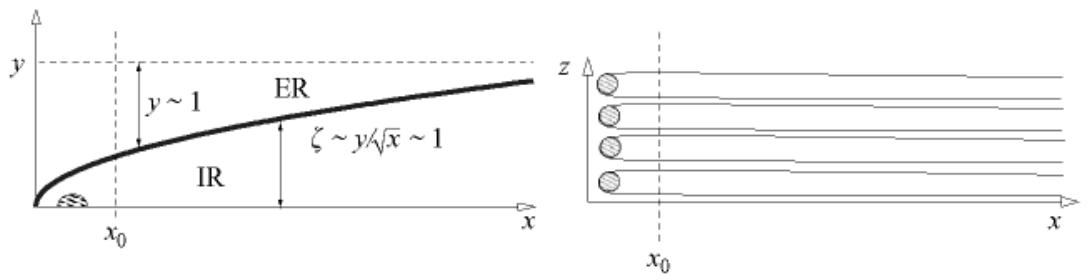

FIGURE 1. Sketch of the boundary layer, with the internal (IR) and external (ER) regions.

Here, we concentrate on freely propagating streaks that are produced somewhere near the leading edge (figure 1), inside the boundary layer. Identifying modes is nontrivial under transient growth. This is in contrast to the case of exponentially growing or decaying eigenmodes, which allow to define/classify modes according to growth intensity. In other words, using the real part of the eigenvalues, modes are classified according to how dangerous (unstable) they are. The counterpart of this criterium in transient growth leads to the somewhat subtle concept of optimal streaks (Andersson et al. 1999; Luchini 2000), which are defined as the maximizers (for varying initial conditions and spanwise wavenumber) of some streamwise energy gain. The objective of this paper is precisely to describe optimal modes through a simpler initial value problem. The main conclusion is that the most dangerous S-perturbations can be considered as just one unstable streaky mode (USM), in the sense that they can be defined in terms of just one streamwise-evolving solution of the linearized equations, with well-defined initial conditions near the leading edge. The latter are calculated solving an eigenvalue problem already considered by Luchini (1996). In other words, this streaky mode is determined by its behaviour near the leading-edge singularity. There are additional modes, but these decay streamwise.

This paper has been strongly inspired by Luchini (1996), Andersson et al. (1999) and Luchini (2000). The remaining of the paper is organized as follows. The linearized problem yielding $S$-perturbations is formulated in $\S 2$, where some previous results are also quoted. The spanwise wavenumber is eliminated from the equations using self-similar variables in $\$ 3$, where the asymptotic behaviours near the free stream and the leading edge are also analysed and a quite efficient numerical scheme to integrate the equations is constructed. All these allow in $\$ 4$ to provide a natural definition of modes and to clarify the structure of optimal perturbations. The paper ends with some concluding remarks, in $\S 5$.

\section{Formulation and other preliminaries}

The starting point is incompressible continuity and Navier-Stokes equations in the usual boundary layer scaling/approximation, assuming a spanwise oscillation with a period comparable to the boundary layer thickness, $\delta^{*}=L^{*} / \sqrt{R e}$, where $L^{*}$ is the distance to the leading edge and $R e=u^{*} L^{*} / \nu \gg 1$ is the Reynolds number based on the free stream velocity $u^{*}$. The streamwise coordinate $x$ is non-dimensionalized with $L^{*}$, the normal and spanwise coordinates with $\delta^{*}$, the streamwise velocity with $u^{*}$, the normal and spanwise components with $u^{*} / \sqrt{R e}$ and the pressure with $\rho^{*}\left(u^{*}\right)^{2}$, as usually. We consider perturbations of a basic, almost parallel, two-dimensional steady state, decompose in normal modes as $(u, v, w, p)=\left(u_{b}, v_{b}, 0, p_{b}\right)+(U, V, \mathrm{i} W, P /$ $R e) \mathrm{e}^{\mathrm{i} \alpha z}+\cdots$ (note that the steady pressure and pressure perturbations scale differently, 
see Luchini 1996) and linearize to obtain

$$
\begin{gathered}
\partial_{x} U+\partial_{y} V-\alpha W=0, \\
\partial_{x}\left(u_{b} U\right)+v_{b} \partial_{y} U+V \partial_{y} u_{b}=\partial_{y y} U-\alpha^{2} U, \\
u_{b} \partial_{x} V+U \partial_{x} v_{b}+\partial_{y}\left(v_{b} V\right)=-\partial_{y} P+\partial_{y y} V-\alpha^{2} V . \\
u_{b} \partial_{x} W+v_{b} \partial_{y} W=-\alpha P+\partial_{y y} W-\alpha^{2} W,
\end{gathered}
$$

where $\partial_{x}, \partial_{y}, \ldots$ denote hereafter partial derivatives. The boundary conditions are

$$
U=V=W=0 \quad \text { at } y=0 \text { and } \propto, \quad P=0 \text { as } y \rightarrow \infty .
$$

We consider a flat plate at zero incidence boundary layer, whose basic steady state is given in (3.1) below and substituted into (2.1)-(2.4) completes the formulation.

The most dangerous perturbation, known as optimal perturbation, was defined by Andersson et al. (1999) and Luchini (2000) as the maximizer of some (kinetic) energy gain between a fixed initial section at $x=x_{0} \gg 1 / \sqrt{R e}$ (to avoid the three-dimensional leading-edge region) and a generic $x$-section. Namely, they maximize the ratio

$$
g=\max \frac{\int_{0}^{\infty}\left[U^{2}+R e^{-1}\left(V^{2}+W^{2}\right)\right] \mathrm{d} y}{\int_{0}^{\infty}\left[\operatorname{Re} U^{2}+\left(V^{2}+W^{2}\right)\right]_{x=x_{0}} \mathrm{~d} y},
$$

for varying $\alpha$ and initial conditions. At large Reynolds number, the $(1 / R e)$-term in the numerator can be neglected. Concerning the denominator, Luchini argues that this should be as small as possible in optimal perturbations and thus he requires that $U=0$ at $x=x_{0}$, which yields a Reynolds-number-independent optimization problem. Andersson et al. instead retained the whole denominator and found optimal perturbations that essentially coincided with those obtained by Luchini and were roughly independent of $R e$ in a range that excluded the limiting value $R e=\infty$. Some remarks about these results are now in order:

(a) Both setting $U=0$ and retaining the whole denominator with finite $R e$ is disturbing because the boundary layer approximation requires that $U^{2} \gg\left(V^{2}+\right.$ $\left.W^{2}\right) / R e$.

(b) As $x \rightarrow 0$, the basic steady state exhibits a characteristic length in the normal direction such that $\zeta=y / \sqrt{x} \sim 1$, which makes the normal coordinate $y$ not appropriate in this limit. But using the variable $\zeta$ does not solve the difficulty either, because the problem also exhibits a second characteristic length, namely $y=\zeta \sqrt{x} \sim 1$, which is associated with decay of the variables to zero to match the outer stream. These two characteristic lengths (see figure 1) will be taken into account below to obtain precise numerical results.

(c) In spite of remarks ( $a$ ) and (b), Andersson et al. (1999) obtained quite robust results using the variable $y$, between the initial and final sections $x_{0} \sim 0.01$ and 1 , which coincided with those by Luchini (2000).

(d) Results showed (figure 5, p. 300 in Luchini 2000) an approximate self-similarity, namely the optimal streamwise velocity scaled with its maximum is approximately constant for varying $\alpha$. An explanation of this was intended by Luchini.

It tums out that the robustness of the results, the approximate self-similarity and the relation between the various definitions of optimal perturbations are related between each other. But understanding all these requires some care. 


\section{The mathematical structure of S-perturbations}

The basic steady state in the Blasius boundary layer is given by

$$
u_{b}=F^{\prime}(\zeta), \quad v_{b}=\left[\zeta F^{\prime}(\zeta)-F(\zeta)\right] /(2 \sqrt{x}), \quad w_{b}=0, \quad p_{b}=-u_{b}^{2} / 2 .
$$

in terms of the self-similar nomal coordinate

$$
\zeta=y / \sqrt{x},
$$

and the streamfunction $F$, given by the Blasius equation $F^{\prime \prime \prime}+F F^{\prime \prime} / 2=0$ in $0<\zeta<\infty$, $F(0)=F^{\prime}(0)=0, F^{\prime}(\infty)=1$, which shows the following asymptotic behaviour at the free stream $(\zeta \rightarrow \infty)$ :

$$
F=\zeta-a+O\left(\mathrm{e}^{-\mathrm{i} \zeta-a !^{2} / 2}\right), \quad \text { with } a \simeq 1.7208 .
$$

3.1. Boundary layer scaling and elimination of the spanwise wavenumber Now, we rewrite (2.1)-(2.5) in terms of the boundary layer variable (3.2). In addition, we use the self-similarity of the linearized equations, inherited from the boundary layer self-similarity, to eliminate the spanwise wavenumber $\alpha$ from the formulation. This is done using (3.2) and the self-similar variables:

$$
\hat{x}=\alpha^{2} x, \quad \hat{V}=\sqrt{x} V, \quad \hat{W}=\sqrt{x} W, \quad \hat{P}=x P,
$$

which allow rewriting $(2.1)-(2.5)$ as

$$
\begin{gathered}
\hat{x} \partial_{\hat{x}} U=\frac{\zeta}{2} \partial_{\zeta} U-\partial_{\zeta} \hat{V}+\sqrt{\hat{x}} \hat{W} \\
\hat{x} F^{\prime} \partial_{\hat{x}} U=\partial_{\zeta \zeta} U+\frac{F}{2} \partial_{\zeta} U+\frac{\zeta F^{\prime \prime}-2 \hat{x}}{2} U-F^{\prime \prime} \hat{V} \\
\hat{x} F^{\prime} \partial_{\hat{x}} \hat{V}=\partial_{\zeta \zeta} \hat{V}+\frac{F}{2} \partial_{\zeta} \hat{V}-\frac{F-\zeta F^{\prime}-\zeta^{2} F^{\prime \prime}}{4} U-\frac{\zeta F^{\prime \prime}-F^{\prime}+2 \hat{x}}{2} \hat{V}-\partial_{\zeta} \hat{P} \\
\hat{x} F^{\prime} \partial_{\hat{x}} \hat{W}=\partial_{\zeta \zeta} \hat{W}+\frac{F}{2} \partial_{\zeta} \hat{W}+\frac{F^{\prime}-2 \hat{x}}{2} \hat{W}-\sqrt{\hat{x}} \hat{P} \\
U=\hat{V}=\hat{W}=0 \quad \text { at } \zeta=0 \text { and } \infty, \quad \hat{P}=0 \text { at } \zeta=\infty
\end{gathered}
$$

\subsection{Behaviour as $\zeta \gg 1$ (external region)}

Invoking (3.3) and noting that the last term in (3.6) decays to zero exponentially fast (as $\exp \left[-(\zeta-a)^{2} / 2\right]$ ) at the edge of the internal region, (3.5)-(3.8) simplify to

$$
\begin{gathered}
U=0, \quad \partial_{\zeta} \hat{V}=\sqrt{\hat{x}} \hat{W} \\
\hat{x} \partial_{\hat{x}} \hat{V}=\partial_{\zeta \zeta} \hat{V}+\frac{\zeta-a}{2} \partial_{\zeta} \hat{V}+\frac{1-2 \hat{x}}{2} \hat{V}-\partial_{\zeta} \hat{P} . \\
\hat{x} \partial_{\hat{x}} \hat{W}=\partial_{\zeta \zeta} \hat{W}+\frac{\zeta-a}{2} \partial_{\zeta} \hat{W}+\frac{1-2 \hat{x}}{2} \hat{W}-\sqrt{\hat{x}} \hat{P} .
\end{gathered}
$$

These correspond (as must be) to the transversal free stream problem obtained linearizing in (2.1)-(2.4) around the steady state $\left(u_{b}, v_{b}, w_{b}\right)=(1, a / 2 \sqrt{x}, 0)$. Proceeding in a standard way (differentiating (3.11) with respect to $\zeta$, multiplying (3.12) by $\sqrt{\hat{x}}$, subtracting and substituting (3.10)), we obtain

$$
\partial_{\zeta \zeta} \hat{P}-\hat{x} \hat{P}=0,
$$

or $\partial_{y y} P-\alpha^{2} P=0$ in original variables. Integration of (3.13), excluding exponentially 
divergent solutions, yields $P=\hat{P}_{\infty}(\hat{x}) \exp [-\sqrt{\hat{x}}(\zeta-a)]$. Substituting this into (3.10)(3.12) and integrating the resulting equations, we obtain

$$
(\hat{V}, \hat{W}, \hat{P})=\left(\hat{V}_{i \infty}(\hat{x}), \hat{W}_{i \infty}(\hat{x}), \hat{P}_{\infty x}(\hat{x})\right) \exp [-\sqrt{\hat{x}}(\zeta-a)],
$$

where $\hat{V}_{\infty}, \hat{W}_{\infty}$ and $\hat{P}_{\infty}$ are such that $\hat{x} \hat{V}_{\infty}^{\prime}=\hat{V}_{\infty} / 2+\sqrt{\hat{x}} \hat{P}_{\infty}$ and $\hat{V}_{\infty}+\hat{W}_{\infty:}=0$. Convergence to the asymptotic values is exponential at the edge of the internal region, namely $(U, \hat{V}, \hat{W}, \hat{P})=\left[\left(0, \hat{V}_{\infty,}, \hat{W}_{\infty,}, \hat{P}_{\infty}\right)+E S T\right] \exp (-\sqrt{\hat{x}}(\zeta-a))$ as $\zeta \rightarrow \infty$, where $E S T$ stands for exponentially small terms, of the order of $\exp \left[-(\zeta-a)^{2} / 2\right]$. This means that the variable

$$
H=\hat{W}+\hat{V}
$$

also behaves as $\exp \left[-(\zeta-a)^{2} / 2\right]$ at large $\zeta$. And invoking (3.14) $H$ is seen to be the transversal divergence in the external region. For convenience, we use this new variable instead of $\hat{W}$, which requires to replace (3.5) and (3.8) by

$$
\begin{aligned}
\hat{x} \partial_{\hat{x}} U= & \frac{\zeta}{2} \partial_{\zeta} U-\partial_{\zeta} \hat{V}-\sqrt{\hat{x}} \hat{V}+\sqrt{\hat{x}} H, \\
\hat{x} F^{\prime} \partial_{\hat{x}} H= & \partial_{\zeta \zeta} H+\frac{F}{2} \partial_{\zeta} H-\frac{F-\zeta F^{\prime}-\zeta^{2} F^{\prime \prime}}{4} U \\
& -\frac{\zeta F^{\prime \prime}}{2} \hat{V}+\frac{F^{\prime}-2 \hat{x}}{2} H-\partial_{\zeta} \hat{P}-\sqrt{\hat{x}} \hat{P} .
\end{aligned}
$$

\subsection{Behaviour as $\hat{x} \ll 1$ (leading edge)}

Assuming that the solution behaves as a power of $\hat{x}$, the approximation as $\hat{x} \rightarrow 0$ is found to be given by an eigenvalue problem. This limit was already considered by Luchini (1996), but the analysis below exhibits two essential differences from Luchini, namely we use the variable $H$ instead of $\hat{W}$ and we take into account the asymptotic behaviour as $\zeta \rightarrow \infty$ (see (3.14) and (3.15)). The asymptotic behaviour is of the form

$$
(U, \hat{V}, H, \hat{P}) \simeq \hat{x}^{-\lambda}(\hat{x} \tilde{U}, \hat{x} \tilde{V}, \sqrt{\hat{x}} \tilde{H}, \hat{x} \tilde{P}) \exp [-\sqrt{\hat{x}}(\zeta-a)],
$$

where, neglecting $O(\sqrt{\hat{x}})$-terms, the various coefficients are given by

$$
\begin{gathered}
\frac{\zeta}{2} \tilde{U}^{\prime}-\check{V}^{\prime}+(\lambda-1) \tilde{U}+\hat{H}=0, \\
\hat{U}^{\prime \prime}+\frac{F}{2} \tilde{U}+\frac{\zeta F^{\prime \prime}+2(\lambda-1) F^{\prime}}{2} \tilde{U}-F^{\prime \prime} \tilde{V}=0, \\
\hat{H}^{\prime \prime}+\frac{F}{2} \hat{H}^{\prime}+\lambda F^{\prime} \hat{H}=0, \\
\tilde{U}=\tilde{V}=\tilde{H}=0 \text { at } \zeta=0, \quad \check{U}, \hat{V}^{\prime}, \tilde{H} \rightarrow 0 \text { as } \zeta \rightarrow \infty .
\end{gathered}
$$

This eigenvalue problem exhibits two kinds of eigenvalues: (i) Luchini eigenvalues $(\lambda=0.7866,1.694,2.627, \ldots$, see Luchini 1996) are given by (3.21) and (ii) Libby-Fox eigenvalues $(\lambda=1,1.887,2.814, \ldots$ see Libby \& Fox 1964) are defined by $(3.19)$ and (3.20), with $\tilde{H}=0$. Note that only the first Luchini mode provides growth in $\hat{x}$. 
Any initial condition, $\left(\hat{U}_{0}, \tilde{V}_{0}, \hat{H}_{0}\right)$, can be written as a series expansion of these modes. The component on each Luchini mode is given by (cf. (13) of Luchini 1996)

$$
a(\tilde{U}, \tilde{V}, \tilde{H}), \quad \text { with } a=\frac{\int_{0}^{\infty}\left(F^{\prime} / F^{\prime \prime}\right) \tilde{H}_{0} \tilde{H} \mathrm{~d} \zeta}{\int_{0}^{\infty}\left(F^{\prime} / F^{\prime \prime}\right) \tilde{H}^{2} \mathrm{~d} \zeta},
$$

as obtained eliminating $\hat{V}$ (upon integrating (3.19) and substituting into (3.20)) to rewrite the whole problem as $\mathscr{L}_{1} \hat{U}+\mathscr{L}_{2} \tilde{H}=0, \mathscr{L}_{3} \hat{H}=0$. The adjoint of this problem is

$$
\mathscr{L}_{1}^{*} \hat{U}^{*}=0, \quad \mathscr{L}_{2}^{*} \hat{U}^{*}+\mathscr{L}_{3}^{*} \hat{H}^{*}=0,
$$

where $\mathscr{L}_{j}^{*}$ stands for the adjoint of the operator $\mathscr{L}_{j}$; note that all these apply independently of the inner product. Since $\lambda$ is not a Libby-Fox eigenvalue, the first equation in (3.24) implies that $\tilde{U}^{*}=0$. Then, (3.19) follows noting that the latter equation is self-adjoint with the inner product $\left\langle\tilde{H}_{1}, \tilde{H}_{2}\right\rangle=\int_{0}^{\alpha}\left(\tilde{H}_{1} \tilde{H}_{2} / F^{\prime \prime}\right) \mathrm{d} \zeta$.

\subsection{Numerics}

As anticipated at the end of $\$ 3.2$, we have replaced (3.5) and (3.8) by (3.16) and (3.17), using the new variable (3.15). In order to integrate the resulting problem in the computational domain $0<\zeta<L$, we note that the basic steady-state converges to its asymptotic value at $\zeta=\infty$ extremely fast, and thus the approximation (3.10)(3.12) applies as (say) $\zeta>12$. Thus, we choose $L_{0}$ such that $12 \leqslant L_{0}<L$, select an equispaced $N$-points mesh in $0<\zeta<L$, and proceed as follows:

(a) Equation (3.7) is replaced by

$$
\partial_{\zeta} \hat{P}+\sqrt{\hat{x}} \hat{P}=0 \quad \text { if } \zeta>L_{0} .
$$

which is equivalent to (3.13) (which, in conjunction with (3.16)-(3.6) and (3.17), is equivalent to (3.7) if $\zeta>L_{0}$ ) if divergent behaviours as $\zeta \rightarrow \infty$ are excluded.

(b) The operator $\partial_{55}$ is discretized using second-order centred differences, $\left(\partial_{\zeta \zeta} q\right)_{n}=\left(q_{n+1}-2 q_{n}+q_{n-1}\right) /\left(2 \delta_{\zeta}^{2}\right)$, with $\delta_{\zeta}=L / N$. The operator $\partial_{\zeta}$ is discretized in (3.5) and (3.25) (which are first order in $\zeta$ ) with second-order forward differences (Lambert 2000), $\left(\partial_{\xi} q\right)_{n}=\left(q_{n-2}-4 q_{n-1}+3 q_{n}\right) /\left(2 \delta_{\zeta}\right)$, and (to avoid upwind instabilities) with second-order backward differences, $\left(\partial_{\zeta} q\right)_{n}=\left(-q_{n+2}+4 q_{n+1}-3 q_{n}\right) /\left(2 \delta_{\zeta}\right)$, in (3.6)-(3.8).

(c) After discretizing spatial derivatives and applying the boundary conditions $U=\hat{V}=H=0$ at $\zeta=0$ and $U=H=0$ at $\zeta=L$ (note that neither the boundary conditions for pressure nor a boundary condition for $\hat{V}$ at $\zeta=L$ are needed), we obtain a system of ordinary differential equations of the form $\hat{x}, \mathscr{H} \partial_{\hat{x}} q=\mathscr{L}(\hat{x}) q$, where $q=(U, \hat{V}, H, \hat{P})$ is the $4 N$ th state vector and $\mathscr{H}$ and $\mathscr{L}$ are $(4 N) \times(4 N)$ matrices. Also, we use the new variable $s=\ln \left(\hat{x} / \hat{x}_{0}\right)$ (which replaces $\hat{x} \partial_{\hat{x}}$ by $\partial_{s}$ ), where $\hat{x}_{0}$ is the initial value of $\hat{x}$.

Marching in $s$ is performed using second-order forward differences, namely

$$
\mathscr{H} \frac{q_{m-2}-4 q_{m-1}+3 q_{m}}{2 \delta_{s}}=\mathscr{L}_{m} q_{m} \text {. }
$$

which are applicable after the second $s$-step; the first step is performed using a first-order forward difference, namely $\left(\partial_{s} q\right)_{1}=\left(q_{1}-q_{0}\right) / \delta_{s}$.

The resulting numerical scheme is extremely efficient and robust because it avoids numerical instabilities and catches well the behaviour as $\zeta \rightarrow \infty$. The latter relies 

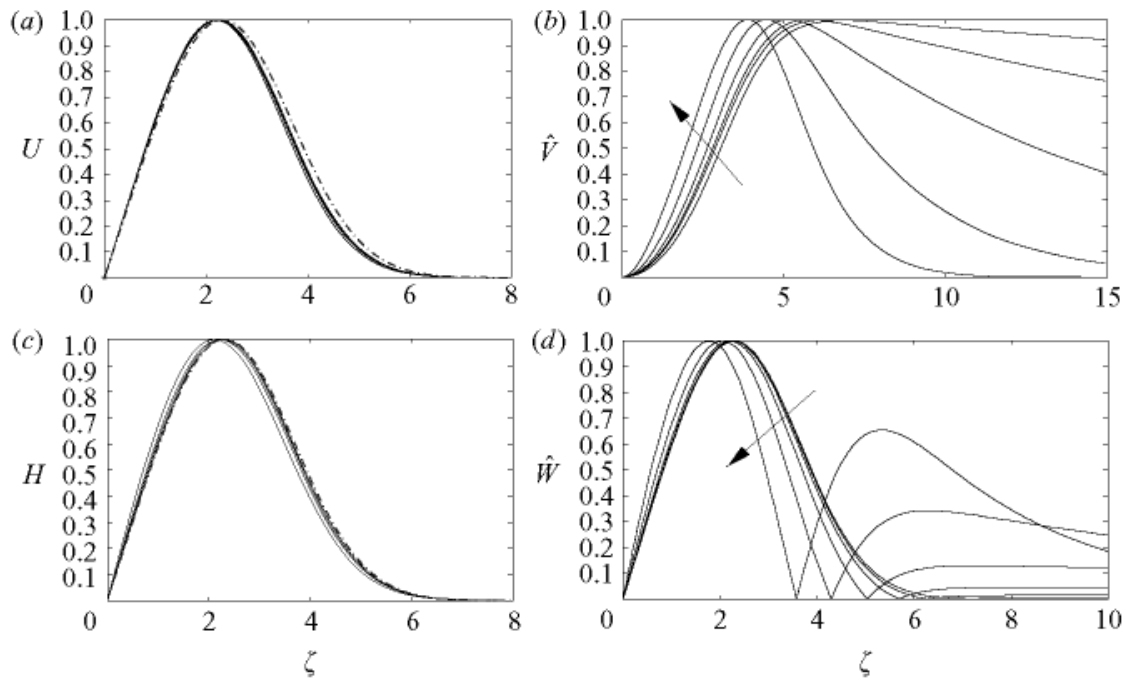

FIGURE 2. Streamwise, cross-flow velocity profiles and $H$-profile, of the USM rescaled with their maxima in $0<\zeta<\infty$, at $\hat{x}=10^{-5}, 10^{-4}, 10^{-3}, 10^{-2}, 10^{-1}$ and 1 ; arrows indicate increasing $\hat{x}$. Luchini's initial conditions, plotted with dashed lines using the same rescaling, are indistinguishable from results at $\hat{x}=10^{-5}$. The streamwise velocity of the Stewartson mode is also shown in $(a)$ and $(c)$ with dot-dashed line.

heavily on (3.25). For instance, integrating in $\hat{x}_{0}=10^{-9}<\hat{x}<1$ (a typical run in next section) with $L=15, L_{0}=12, N=150$ and $500 \mathrm{~s}$-steps (which means that $\delta_{\zeta}=0.1$ and $\left.\delta_{s}=9 \ln (10) / 500=0.0461\right)$ only requires $50 \mathrm{CPU}$ seconds using MATLAB in a standard desktop computer. Results are exact within plot accuracy and independent of $L, L_{0}, \delta_{\zeta}$ and $\delta_{s}$ provided that $12 \leqslant L-3 \leqslant L_{0} \ll L, \delta_{\zeta} \leqslant 0.1, \delta_{s} \leqslant 0.05$ and $0.2 \leqslant \delta_{\zeta}^{2} / \delta_{s} \leqslant 5$. The latter requirement comes from the fact that $\delta_{\zeta}^{2} / \delta_{s}$ should be neither too small nor too large because (3.26) is a singular implicit system, namely $\mathscr{M}$ and $\mathscr{L}_{m}$ are both singular.

\section{Results}

The evolution equations (3.5)-(3.7), (3.16)-(3.17) must be completed with initial conditions. To begin with, we take as initial condition at $\hat{x}_{0}=10^{-9}$ the first Luchini mode, as defined in (3.18). The resulting solution, rescaled with the maximum of $|U|$ in $\hat{x}_{0}<\hat{x}<1,0<\zeta<\infty$, will be called the USM below and denoted as $\left(U^{u}, \hat{V}^{u}, H^{u}, \hat{P}^{u}\right)$. The streamwise, cross-flow velocity profiles and the $H$-profile (normalized with their maxima in $0<\zeta<\infty$ ) are plotted in figure 2, and the maxima of $U, \hat{V}, \hat{W}$ and $H$ are plotted versus $\hat{x}$ with solid lines in figure $3(a)$. As already noticed by Luchini (2000), figure 2(a) shows that the streamwise velocity of the optimal perturbation remains approximately constant, up to rescaling, and approximately equal to both the streamwise velocity of the first Libby-Fox mode, $U=\zeta F^{\prime \prime}$ (Stewartson 1957; Libby \& Fox 1964; and Luchini 1996) and the streamwise velocity of the first Luchini mode; these two are also plotted for comparison. Figure 2 also shows that the new variable $H$ introduced above also remains approximately constant and approximately equal to $U$ (when rescaled with its maximum), which suggests that a low-dimensional Ordinary Differential Equation (ODE) model of streaks should be possible. 

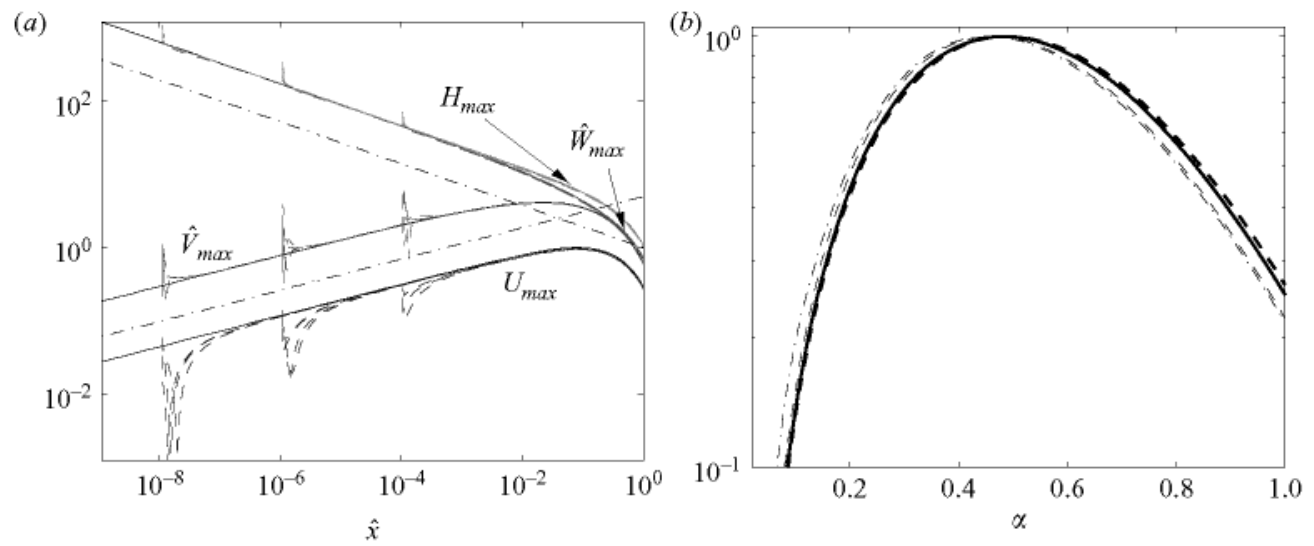

Figure 3. (a) Maxima (in $0<\zeta<\infty$ ) of $U, \hat{V}, H$ and $\hat{W}$ versus $\hat{x}$ using random initial conditions at $\hat{x}_{0}=10^{-8}, 10^{-6}$ and $10^{-4}$ (dashed lines) and using the projection on Luchini's initial conditions (solid lines); the asymptotic behaviours (see (3.18)) $\hat{W} \sim H \sim \hat{x}^{1 / 2-\lambda_{1}}$ and $\hat{V} \sim \hat{x}^{1-\lambda_{1}}$, with $\lambda_{1}=0.7866$, are plotted with dot-dashed lines. (b) Maximum of 'energy gain' $G^{L}$ (plotted versus the spanwise wavenumber) between $x=0.01$ and $x=1$ (thin dot-dashed) and between $x=10^{-5}$ and 1 (thin solid); the quotient $G^{L}$ calculated on the USM between $x=0.01$ and $x=1$ (thin dashed); the quotient $G^{u}$ calculated over the USM between $x=0.01$ and $x=1$ (thick dashed) and between $x=10^{-5}$ and 1 (thick solid). In order to facilitate comparison between the various curves, all of them are normalized with their maxima in $0<\alpha<1$.

In addition, in order to illustrate the effect of general initial conditions, we consider initial conditions of the form $(U, \hat{V}, H, \hat{P})=\left(\sqrt{\hat{x}_{0}} U_{0}, \sqrt{\hat{x}_{0}} \hat{V}_{0}, H_{0}, \sqrt{\hat{x}_{0}} \hat{P}_{0}\right)$, which scale with the boundary layer scaling (3.18). Here, we set $\hat{V}_{0}=\hat{P}_{0}=0$ and choose $U_{0}$ and $H_{0}$ randomly as follows

$$
U_{0}=\left[\sum_{k=0}^{4} \gamma_{k}^{1} \cos (k \zeta)\right] \tilde{U}_{L}, \quad H_{0}=\left[1+\sum_{k=1}^{4} \gamma_{k}^{2} \cos (k \zeta)\right] \tilde{H}_{L},
$$

where $\tilde{U}_{L}$ and $\tilde{H}_{L}$ are the $U$ and $H$ components of the first Luchini eigenfunction and $\gamma_{k}^{1}$ and $\gamma_{k}^{2}$ are chosen randomly in the interval between -1 and 1 . Fixing two of the four variables makes sense since the initial conditions should satisfy two compatibility conditions (which are selected by the equations after a few integration steps if not satisfied initially). One of these is obtained multiplying (3.16) by $F^{\prime}$ and subtracting (3.6), and the other one by substituting equations (3.6)-(3.17) into the $\hat{x}$-derivative of (3.16). The result is plotted (after rescaling as indicated below) with dashed lines in figure $3(a)$. Now, for each of these initial conditions, we consider its projection (defined according to (3.23)) on the USM at this value of $\hat{x}_{0},\left(U_{0}^{u}, \hat{V}_{0}^{u}, H_{0}^{u}, \hat{P}_{0}^{u}\right)=\left(U^{u}\left(\hat{x}_{0}\right), \hat{V}^{u}\left(\hat{x}_{0}\right), H^{u}\left(\hat{x}_{0}\right), \hat{P}^{u}\left(\hat{x}_{0}\right)\right)$, namely

$$
\left(\tilde{U}_{0}, \tilde{V}_{0}, \tilde{H}_{0}, \tilde{P}_{0}\right)^{\text {proj }}=a\left(U_{0}^{u}, \hat{V}_{0}^{u}, H_{0}^{u}, \hat{P}_{0}^{u}\right), \quad \text { with } a=\frac{\int_{0}^{\infty}\left(F^{\prime} / F^{\prime \prime}\right) H_{0} H_{0}^{u} \mathrm{~d} \zeta}{\int_{0}^{\infty}\left(F^{\prime} / F^{\prime \prime}\right)\left(H_{0}^{u}\right)^{2} \mathrm{~d} \zeta} .
$$

Thus, the projected initial conditions yield a solution that is proportional to the USM; for comparison, the original solution is rescaled such that the solution with projected initial conditions exactly coincides with the USM. These initial conditions are applied 
several times at various values of $\hat{x}_{0}$ (namely, at $\hat{x}_{0}=10^{-8}, 10^{-6}$ and $10^{-4}$ ), obtaining the solutions plotted with dashed lines in figure $3(a)$. Note that:

(a) After an initial streamwise transient, all solutions approach the USM up to a constant amplitude. Equation (4.2) allows us to calculate the amplitude of the USM for arbitrary initial conditions. It is precisely in this sense that the USM is completely similar to what are called modes in equations with constant coefficients.

(b) Any initial condition can be expanded into the (infinitely many) eigenmodes considered in $\$ 3.3$, to obtain a complete system of initial conditions, which provides a complete system of solutions; the first of these, namely that associated with the first Luchini eigenvalue, is the USM and is the only one that does not decay as $\hat{x}$ grows. This means that for sufficiently small $x_{0}$ all these solutions contribute to the denominator in (2.6) but only the USM contributes to the numerator, which means that the maximizer of (2.6) must be precisely the USM.

(c) Thus transients in figure 3 are associated with the projection of the initial condition on the remaining modes considered in $\$ 3.3$. Since the most dangerous of these is the second Luchini mode, which decays as $\left(\hat{x}_{0} / \hat{x}\right)^{\hat{x}_{2}-\hat{\lambda}_{1}} \sim\left(\hat{x}_{0} / \hat{x}\right)^{0.907}$, transients survive until (say) $\hat{x} / \hat{x}_{0} \sim 10$ if the initial amplitude of the second Luchini mode is comparable to that of the first mode; if the former is much larger than the latter, transients will enlarge. This is consistent with the experiments by Fransson et al. (2004), which showed remarkable agreement with the linear theory whenever $x / x_{0}=\hat{x} / \hat{x}_{0}$ were larger than 10. Precise description of other experiments in this paper, with $x / x_{0}=5,1.75$ and 1.375 , would require to consider more modes.

(d) In order to compare with Luchini's results, we consider the following expressions

$$
G^{L}=\frac{\sqrt{x_{0}}\left[\int_{0}^{\infty_{1}} U^{2} \mathrm{~d} \zeta\right]_{\dot{i}=\alpha^{2}}}{\left[\int_{0}^{\alpha_{x}}\left(\hat{V}^{2}+\hat{W}^{2}\right) \mathrm{d} \zeta\right]_{\dot{x}=x_{0} \alpha^{2}}}, \quad G^{u}=\frac{\sqrt{x_{0}}\left[\int_{0}^{\infty_{i}} U^{2} \mathrm{~d} \zeta\right]_{\dot{x}=\alpha^{2}}}{\left[\int_{0}^{\infty}\left(F^{\prime} / F^{\prime \prime}\right) H^{2} \mathrm{~d} \zeta\right]_{\dot{x}=x_{0} \alpha^{2}}} .
$$

Here, $G^{L}$ is (invoking (3.4)) precisely the kinetic energy gain maximized by Luchini (2000), namely the quotient (2.6), with $R e^{-1}=0$ in the numerator and $R e=0$ in the denominator. The maximum of this quotient for varying initial conditions at $x_{0}=0.01$ (calculated by Luchini) is plotted with thin dot-dashed line in figure $3(b)$; the maximum of this curve is attained at $\alpha=0.45$. For comparison, the quotient $G^{L}$ calculated along the USM is plotted with thin dashed line. The difference of both curves is just due to the effect of the remaining modes in the maximization process, which are not negligible at $x_{0}=0.01$. At smaller $x_{0}$ both curves coincide, which is illustrated at $x_{0}=10^{-5}$ (solid thick curve); the maximum of this curve is attained at $\alpha=0.484$, which is the asymptotic value of Luchini's optimal wavenumber. The second quotient in (4.3) corresponds to substituting the denominator in Luchini by the square of the initial amplitude of the USM, as defined in (4.2). The quotient $G^{\mu}$ along the USM for $x_{0}=10^{-5}$ and $x_{0}=0.01$ are plotted in figure 3 with thick-solid line and thickdashed lines, respectively; note that the former coincides with its counterpart for $G^{L}$.

\section{Concluding remarks}

We believe that the results above clarify the structure of internal streaky perturbations in Blasius boundary layers. In particular, we have shown that this structure is quite simple and qualitatively similar to standard eigenmodes in standard linear stability theory. The only differences are that growth is algebraic instead of 
exponential and that the streamwise evolution exhibits a growth-decay shape. The consequence is that the optimization procedure used so far to define optimal streaks is not necessary; modes instead are defined in terms of a well-defined evolution problem. with well-defined initial conditions. The results above relied on two main ingredients, namely self-similarity of the boundary layer allowed to eliminate the wavenumber from the formulation and our quite robust numerical scheme (based on a careful analysis of the behaviour of the solutions in the external region, which showed that this is slaved to the internal region) and thus allowed to obtain precise results with initial conditions at a section extremely close to the leading edge. Several additional consequences of the results of the paper are in order:

(a) We expect that other self-similar two-dimensional boundary layers subject to three-dimensional internal streaky perturbations be amenable to similar treatment. This point is currently under research.

(b) Self-similarity is not present in general two-dimensional boundary layers, but we expect that internal perturbations of these still show a behaviour in the external region that is slaved to the behaviour in the internal region; thus, an efficient numerical scheme should also be possible.

(c) The fact that forcing resulted only from internal perturbations, occurring near the leading edge was essential in the analysis above. External forcing from perturbations in the free stream produces a different behaviour in the external region (namely, the velocity components do not decay to zero) and thus requires a different treatment. This point is currently under research.

(d) Internal initial perturbations may result, for instance, from arrays of threedimensional objects near the leading edge (figure 1). We expect that the resulting threedimensional flow relaxes after several boundary layer thicknesses to a velocity profile that exhibits the scaling encountered in $\$ 3.3$, which can be taken as a streamwise initial condition to the equations considered above; but according to our results in $\$ 4$, the velocity profiles evolve streamwise fairly fast to the unstable streaky mode, which dominates downstream. Thus, the effect of the details of the leading-edge obstacles should be appreciated only in the amplitude of the unstable streaky mode.

This research was partially supported by the Spanish Ministry of Education, under Grant TRA2007-65699. We are indebted to two anonymous referees for some useful comments that helped to improve presentation of the results.

\section{REFERENCES}

Andersson. P., Berggren, M. \& Henningson, D. S. 1999 Optimal disturbances and bypass transition in boundary layers. Phys. Fluids 11, 134-150.

Bagget, J. S. \& Threfethen, L. N. 1997 Low dimensional models of subcritical transition to turbulence. Phys. Flidis 9, 1043-1053.

Cossu. C. \& BRANDT, L. 2002 Stabilization of Tollmien-Schlichting waves by finite amplitude optimal streaks in a Blasius boundary layer. Phys. Fluids 14, L.57-L.60.

Crow, S. C. 1966 The spanwise perturbations of two dimensional boundary layers. J. F/uid Mech. 24 153-164.

Ellingsen, T. \& Palm. E. 1975 Stability of linear flow. Phys. Fluids 18, 487-488.

Fransson, J. H. M.. Brandt, L., Talamelli, A. \& Cossu, C. 2004 Experimental and theoretical investigation of the nonmodal growth of steady streaks in a flat plate boundary layer. Phys. Fiuds 16, 3627-3638.

Fransson, J. H. M., Talamelli, A., Brandt, L. \& Cossu, C. 2006 Delaying transition to turbulence by a passive mechanism. Phis. Rev. Lett. 96, 064501-1-4. 
HuligRen, L. S. \& Gustavsson, L. H. 1981 Algebraic growth of disturbances in a laminar boundary layer. Phys. Fluids 24, 1000-1004.

Klebanoff, P. S., Tidstrom. K. D.. \& Sargent, L. M. 1962 The three-dimensional nature of boundary layer instability. $J$. Fluid Mech. 12, 1-34.

LAMBERT, J. D. 2000 Nimerical Methods for Ordinary Differential Systems. John Wiley \& Sons.

LANDAHL, M. T. 1980 A note on the algebraic instability of inviscid parallel shear flows. J. Fluid Mech. 98, 243-251.

Leib, S. J., Wundrow, D. W. \& Goldstein. M. E. 1999 Effect of free-stream turbulence and other vortical disturbances on laminar boundary layer $J$. Fluid Mech. 380, 169-203.

LibBY, P. A. \& Fox, H. 1964 Some perturbation solutions in laminar boundary-layer theory. J. Fluid Mech. 17, 433-449.

LuchINI, P. 1996 Reynolds-number-independent instability of boundary layer over a flat surface. J. Fluid Mech. 327, 101-115.

LUCHINI, P 2000 Reynolds-number-independent instability of boundary layer over a flat surface: optimal perturbations. J. Flitid Mech. 404, 289-309.

Morkovin, M. V. 1984 Bypass transition to turbulence and research desiderata. In LaminarTurbulent Transition (ed. D. Arnal \& R. Michel), pp. 3-29. Springer.

Morkovin. M. V. \& Reshotкo. E. 1990 Dialog on progress and issues in stability and transition research. In Tratsition in Turbities, pp. 161-204. NASA Conf. Pub. 2386.

Schmid, P. J. 2007 Nonmodal stability theory. Anmu. Rev. Fluid Mech. 39, 129-162.

STEWARTSON, K. 1957 On asymptotic expansion in the theory of laminar boundary layer. $J$. Math. Phys. 36, 137-191.

Threfethen, L. N., Threfethen, A. E., Reddy, S. C. \& Driscoll, T. A. 1993 Hydrodynamic stability without eigenvalues. Science 261, 578-584. 